\title{
Invaginación del apéndice: un hallazgo poco frecuente en colonoscopia. Informe de caso
}

\section{Appendiceal intussusception: a rare finding during colonoscopy. Case report}

\author{
Luis Jorge Lombana-Amaya, ${ }^{{ }^{*}}$ (1) Saúl Rugeles, ${ }^{2}$ (1) Jorge Alejandro González-González. ${ }^{3}$
}

\author{
Gacceso abierto \\ Citación: \\ Lombana-Amaya LJ, Rugeles S, González- \\ González JA. Invaginación del apéndice: un \\ hallazgo poco frecuente en colonoscopia. \\ Informe de caso. Rev Colomb Gastroenterol. \\ 2021:36(2):275-279. https://doi. \\ org/10.22516/25007440.638 \\ MD, profesor de cirugía, Universidad \\ Javeriana. Jefe de la unidad de colon y recto, \\ Departamento de Cirugía, Hospital Universitario \\ San Ignacio. Bogotá, Colombia. \\ 2 MD, profesor de cirugía, Universidad Javeriana. \\ Cirujano gastrointestinal, Departamento de \\ Cirugía, Hospital Universitario San Ignacio. \\ Bogotá, Colombia. \\ $3 \mathrm{MD}$, residente de cirugía general, Departamento \\ de Cirugía, Hospital Universitario San Ignacio, \\ Facultad de medicina, Pontificia Universidad \\ Javeriana. Bogotá, Colombia. \\ *Correspondencia: Luis Jorge Lombana-Amaya \\ ljlombana@hotmail.com \\ Fecha recibido: $\quad 13 / 08 / 20$ \\ Fecha aceptado: 02/09/20
}

\begin{abstract}
Resumen
Se presenta el caso de una mujer joven con hallazgo endoscópico incidental en una colonoscopia de seguimiento, que consistía en una lesión en el ciego con histología benigna. Se describe el proceso diagnóstico y el tratamiento quirúrgico. Se revisa la literatura existente y se discuten la incidencia, el cuadro clínico y las indicaciones de tratamiento quirúrgico de una patología rara de baja aparición.
\end{abstract}

\section{Palabras clave}

Intususcepción, neoplasia apendicular, colonoscopia, endometriosis.

\section{Abstract}

This is the case of a young patient with an incidental endoscopic finding of a lesion in the cecum during follow-up colonoscopy with benign histology. The diagnostic process and surgical treatment are described. The existing literature was reviewed and the incidence, symptoms and indications of surgical treatment of this rare condition are discussed.

\section{Keywords}

Intussusception; Appendiceal neoplasm; Colonoscopy; Endometriosis.

\section{INTRODUCCIÓN}

La intususcepción apendicular está definida como la invaginación del apéndice o una porción del mismo y parte de mesenterio correspondiente dentro de la luz del intestino contiguo. El primer caso reportado en la literatura fue un paciente de 7 años de edad en 1858 por McKidd (1). Es una entidad poco común y cuenta con escasos reportes en la literatura, con una incidencia del 0,01 \% según un estudio de cohortes realizado por Collins, que contó con 71000 pacientes llevados a apendicectomía en un período de 40 años $(2,3)$. Es más frecuente en mujeres hacia la cuarta o quinta décadas de la vida. El diagnóstico de esta entidad es compleja, debido a su clínica no específica y su baja incidencia. El pro- 
pósito de esta publicación es presentar un caso tratado en nuestra institución.

\section{REPORTE DE CASO}

Se trata de una mujer de 43 años con antecedente de un tumor neuroendocrino de bajo grado a nivel del recto superior, resecado por endoscopia 3 años antes, quien se encontraba en seguimiento clínico y endoscópico. Era completamente asintomática y en la última colonoscopia total se encontró una lesión a nivel del meato apendicular de $2 \mathrm{~cm}$ (Figura 1). Se realizó una biopsia de la lesión con reporte de mucosa de aspecto sano sin hallazgos que sugirieran malignidad u otros cambios patológicos. Una tomografía de abdomen y pelvis, y los niveles de ácido 5-hidroxindolacético fueron normales. El examen clínico fue normal.

Aunque el estudio histopatológico era no conclusivo, se decidió dar manejo quirúrgico por mínima invasión. Se inició la cirugía con una laparoscopia en la que se encontró ausencia de apéndice cecal; se practicó entonces una incisión de Rockey Davis usando un retractor de piel tipo Alexis para exponer el ciego. A la palpación y visión se confirmó una invaginación apendicular (Figura 2).

Se realizó una cecectomía con sutura mecánica lineal cortante y se envió el espécimen a patología (Figura 3). La evolución postoperatoria fue adecuada y sin complicaciones. En patología se confirmó la invaginación secundaria a focos de endometriosis (Figura 4).

En controles posoperatorios se logró obtener el informe de colonoscopia de hace 3 años cuando se manejó el de tumor neuroendocrino en el recto, con un reporte del ciego y meato apendicular dentro de los límites normales, aunque sin fotodocumentación.

\section{DISCUSIÓN}

La fisiopatología de esta entidad no es clara; sin embargo, está asociada con la presencia de alteraciones apendiculares, principalmente con la existencia de masa a ese nivel. Se cree que se presenta peristaltismo alterado, y se encuentra asociado con inflamación, cuerpo extraño, enfermedad de Crohn, parásitos, pólipos, hiperplasia linfoide, hematoma, linfoma de tejido linfoide asociado a mucosas (MALT), adenoma, papiloma, adenocarcinoma, endometriosis y mucocele (4-7); estas 2 últimas son las causas principales en adultos, mientras que los procesos inflamatorios son los más comunes en edad pediátrica (8-10).

Dada su baja incidencia, clínica variada y poco específica, el diagnóstico de esta entidad implica un reto para el médico. Se han descrito 3 tipos de sintomatología en estos pacientes:

- el paciente que cursa con síntomas de apendicitis aguda típica;

- síntomas de intususcepción (dolor abdominal grave, vómito, diarrea y melenas) que son inespecíficos; y

- sensación de masa, dolor en el cuadrante inferior derecho y melenas de varios meses de evolución.

La mayoría de los pacientes son asintomáticas con hallazgo incidental en un estudio colonoscópico, también se puede encontrar como hallazgo intraoperatorio en una cirugía $(57 \%)$ o por parte de patología $(11 \%)(1,7,8,11)$.
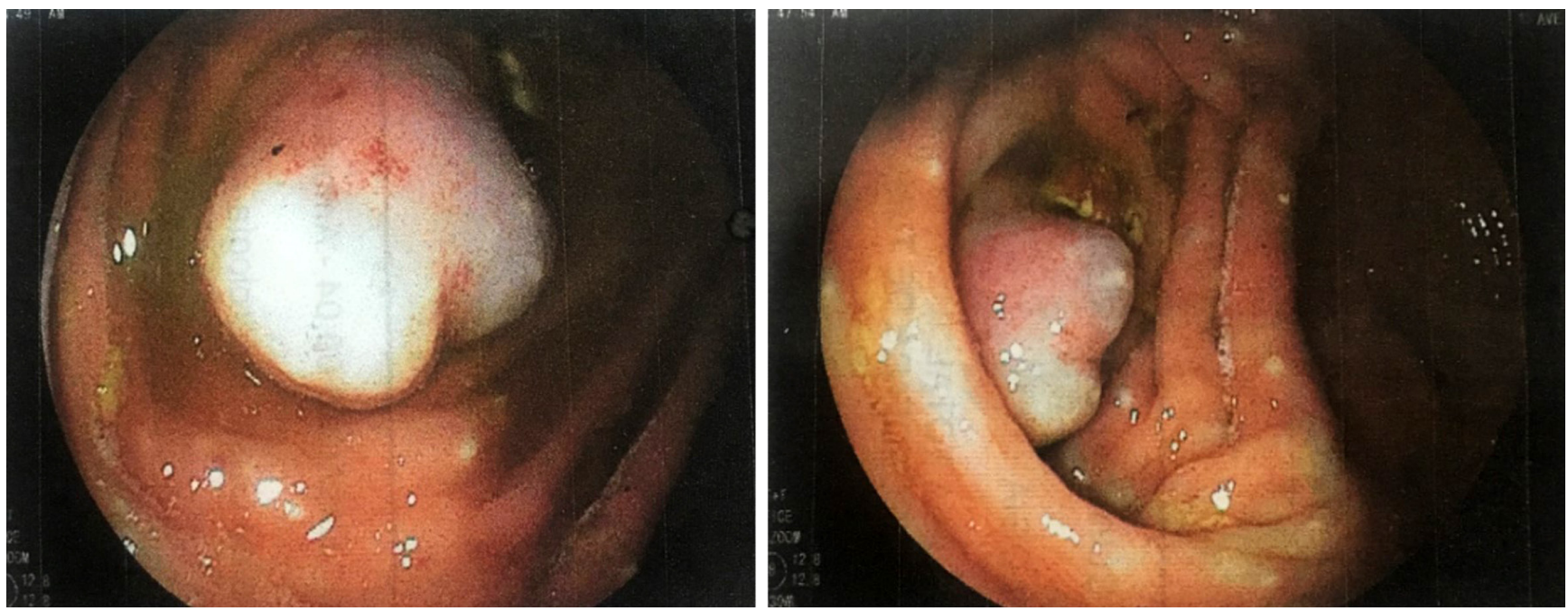

Figura 1. Apéndice cecal invaginado, visión de la colonoscopia en el polo cecal. 


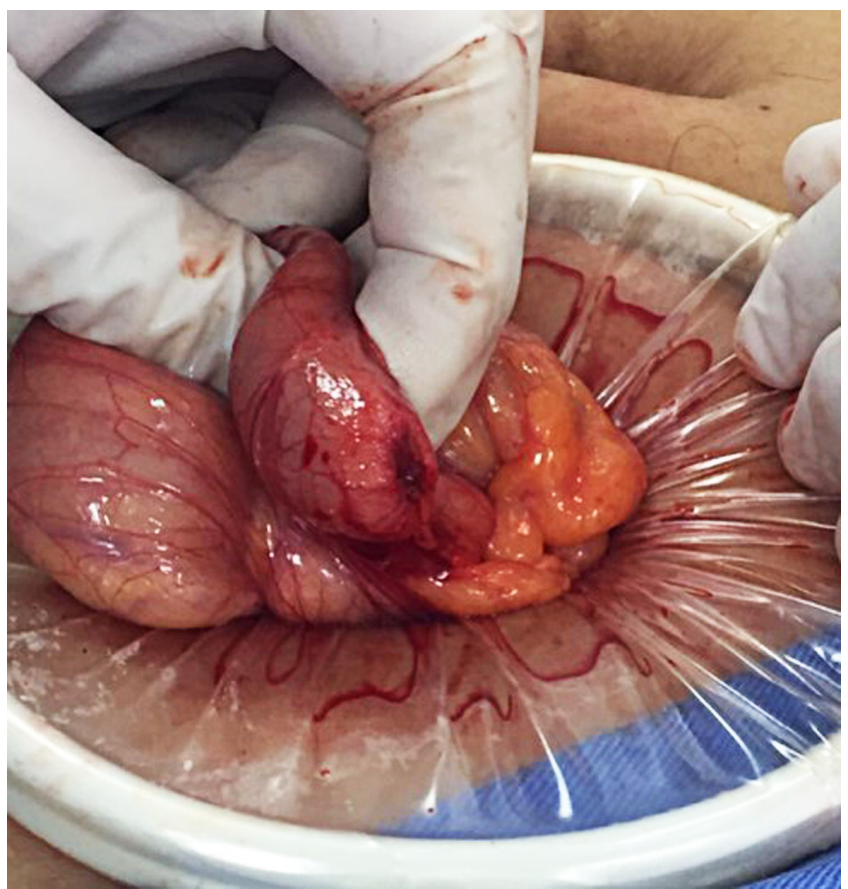

Figura 2. Ciego sin apéndice por invaginación, hallazgo quirúrgico.

En cuanto a la etiología, la causa más común de intususcepción apendicular en adultos es la endometriosis, una entidad benigna descrita por primera vez en 1860 por Von Rokitansky (12). Se define como la presencia de glándulas y estroma endometrial fuera de la cavidad uterina. La endome- triosis afecta a las mujeres en un $10 \%$ a $15 \%(11,13-15)$. El recto y el sigmoide son los principales sitios de implantación intestinal, seguidos por colon ascendente, intestino delgado y el apéndice, que abarca del $0,08 \%$ al $8 \%$ de los casos (Figura 5) $(8,10,12,16)$.

Histológicamente, se caracteriza por la presencia de glándulas y estroma tipo endometrioide, que puede estar asociado con macrófagos. Dentro de los cambios benignos atípicos se pueden encontrar nódulos pseudoxantomatosos necróticos; afección vascular, perineural y ganglionar; y cambios metaplásicos tanto en las glándulas como en el estroma. Además, puede contener cambios premalignos como endometriosis polipoide, endometriosis estromal, hiperplasia mesotelial asociado con endometriosis y endometriosis atípica con un riesgo de conversión a cáncer del $1 \%(15)$.

Dentro de los métodos diagnósticos utilizados se encuentran la tomografía de abdomen contrastada y el colon por enema, seguido de la ecografía, que tiene una gran utilidad en la población pediátrica. En la colonoscopia en adultos realizada por diferentes indicaciones se llega a describir como un pólipo pseudopediculado (17-19), o masa a nivel del meato apendicular, como fue en este caso. No se han reportado más de 200 casos en la literatura; sin embargo, es imposible conocer la incidencia de dicho hallazgo. Si se enfoca como lesión polipoide, la realización de una polipectomía puede generar el riesgo de perforación y peritonitis (20). Es importante pensar que puede existir esta entidad cuando se evalúen lesiones a nivel del meato apendicular. Un reporte de McSwain planteó una clasificación de la
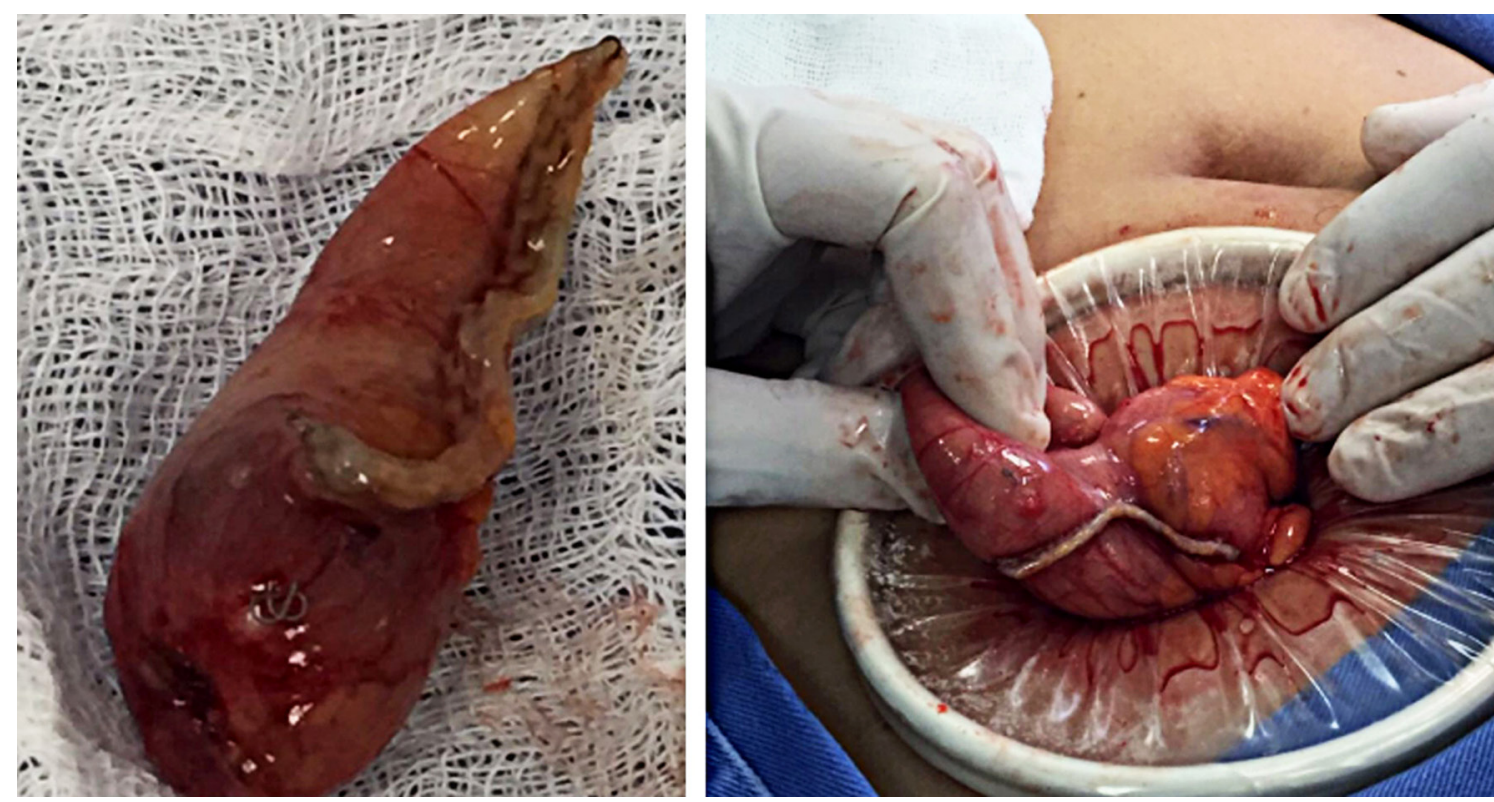

Figura 3. Cecectomía con sutura mecánica. 


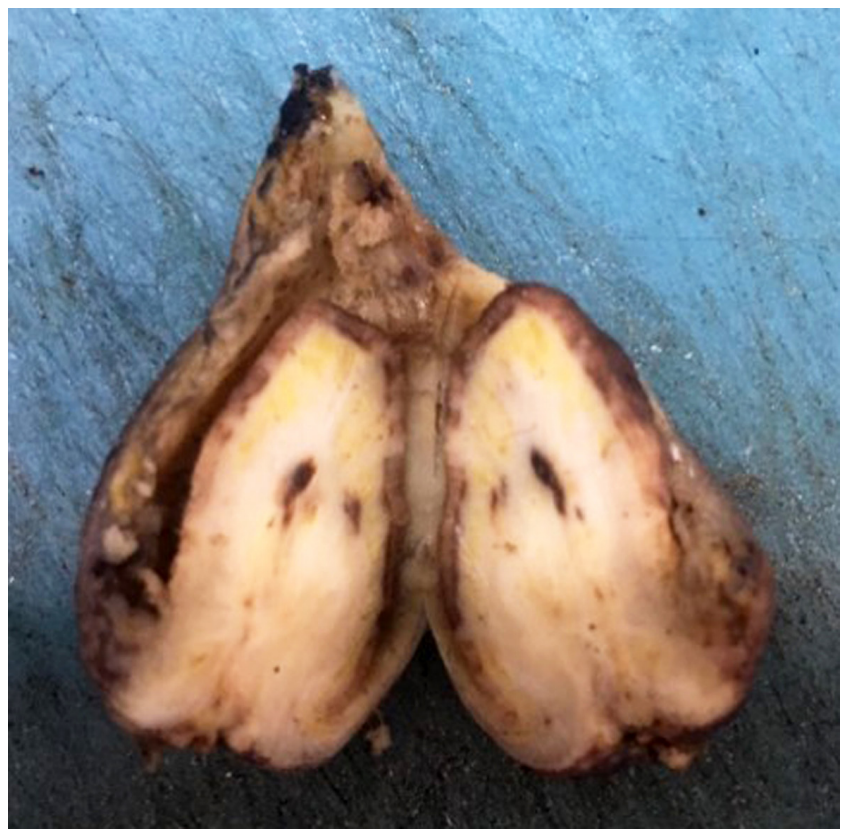

Figura 4. Pieza de patología que evidencia del apéndice completo invaginado y su luz.

intususcepción dependiendo de la anatomía encontrada, simplificada años después por Langsman $(17,21)$ :
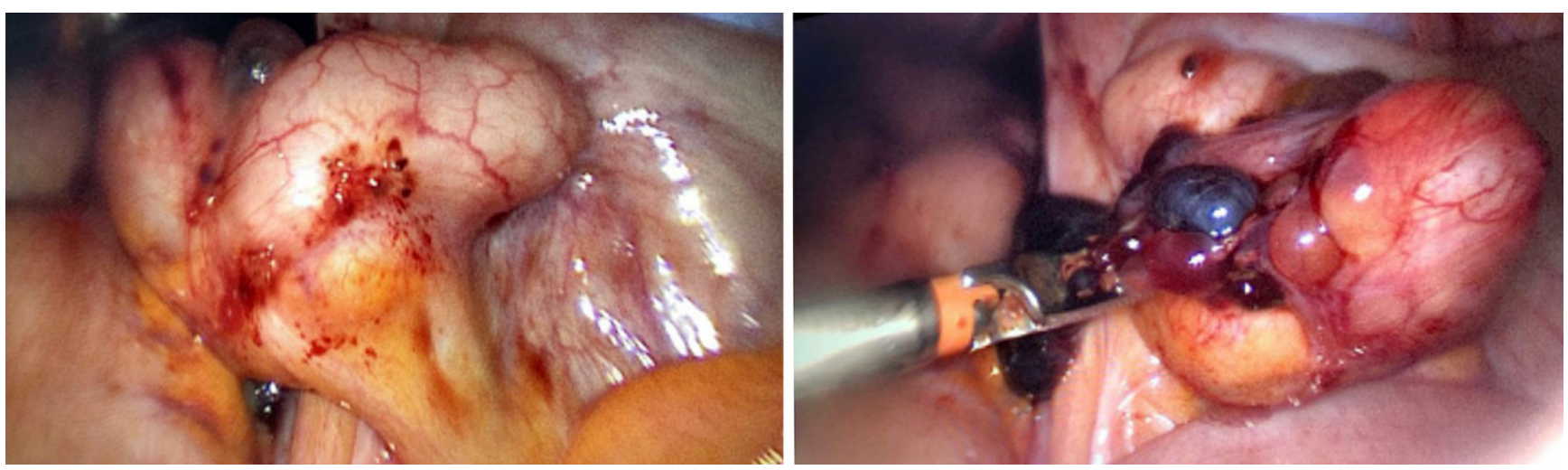

- tipo I: invaginación de la punta del apéndice sobre la porción proximal del apéndice,

- tipo II: la intususcepción se generada en algún punto a lo largo del apéndice diferente a la punta y la base del mismo,

- tipo III: inicio de la intususcepción a nivel de la base apendicular que se introduce dentro de la parte más distal del ciego,

- tipo IV: invaginación retrógrada de un segmento proximal dentro de un segmento distal del mismo apéndice,

- tipo V: invaginación completa del apéndice en el ciego (este caso).

El tratamiento quirúrgico es el manejo estándar, teniendo en cuenta que siempre existe la probabilidad de patología maligna como causante de la invaginación. En la gran mayoría de los casos, los pacientes son llevados a cecectomía y, en algunos casos, a hemicolectomía derecha (22). La endometriosis es la mayor causa descrita en los reportes de los últimos 10 años.

Este caso ilustra muy bien la patología: en los 2 años posteriores en el servicio se han realizado dos cecectomías más con invaginación apendicular secundaria a endometriosis, lo cual sugiere que la frecuencia no es tan baja como se cita en la literatura. Por esta razón, la intususcepción apendicular debe figurar como uno de los diagnósticos diferenciales de las lesiones del meato apendicular.

Figura 5. Focos de endometriosis comprometiendo un apéndice cecal.

\section{REFERENCIAS}

1. McKidd J. Case of invagination of caecum and appendix. Edinburgh Med J. 1858;4:793-7.

2. Chaar CI, Wexelman B, Zuckerman K, Longo W. Intussusception of the appendix: comprehensive review of the literature. Am J Surg. 2009;198(1):122-8.

https://doi.org/10.1016/j.amjsurg.2008.08.023 
3. Collins DC. 71,000 human appendix specimens. A final report, summarizing forty years' study. Am J Proctol. 1963;14:265-81.

4. Atkinson GO, Gay BB Jr, Naffis D. Intussusception of the appendix in children. AJR Am J Roentgenol. 1976; 126(6): 1164-8. https://doi.org/10.2214/ajr.126.6.1164

5. Gilpin D. Intussusception of the appendix. Ulster Med J. 1989;58(2):193-5.

6. Komine N, Yasunaga C, Nakamoto M, Shima I, Iso Y, Takeda Y, et al. Intussusception of the appendix that reduced spontaneously during follow-up in a patient on hemodialysis therapy. Intern Med. 2004;43(6):479-83. https://doi.org/10.2169/internalmedicine.43.479

7. Tăban S, Dema A, Lazăr D, Sporea I, Lazăr E, Cornianu M. An unusual «tumor» of the cecum: the inverted appendiceal stump. Rom J Morphol Embryol. 2006;47(2):193-6.

8. Lee DJ, Kim HC, Yang DM, Kim SW, Ryu JK, Won KY, et al. A case of intussusception of the appendix secondary to endometriosis: US and CT findings. J Clin Ultrasound. 2015;43(7):443-6. https://doi.org/10.1002/jcu.22188

9. Duncan JE, DeNobile JW, Sweeney WB. Colonoscopic diagnosis of appendiceal intussusception: case report and review of the literature. JSLS. 2005;9(4):488-90.

10. Liang HH, Huang MT, Wei PL, Weu W, Lin YH, Tiang C, et al. Endometriosis-induced appendiceal intussusception. Am J Surg. 2009; 197(6):e66-8. https://doi.org/10.1016/j.amjsurg.2008.04.024

11. Sánchez Santamaría M, Sánchez Gualberto A, Alcóucer Díaz MR, Creagh Cerquera R, Pujol de la Llave E. Endometriosis como causa de invaginación intestinal. Rev Clin Esp. 2007;207(10):536-7. Spanish. https://doi.org/10.1157/13111561

12. Costa M, Bento A, Batista H, Oliveira F. Endometriosisinduced intussusception of the caecal appendix. BMJ Case Rep. 2014;2014:bcr2013200098. https: / doi.org/10.1136/bcr-2013-200098

13. Pérez Cabanas I, Zornoza G, Arconada FA, Oroz F, Voltas F. Endometriósis del apéndice vermiforme. Rev Med Univ Navarra. 1974;18(1-2):69-76.
14. Gustofson RL, Kim N, Liu S, Stratton P. Endometriosis and the appendix: a case series and comprehensive review of the literature. Fertil Steril. 2006;86(2):298-303. https://doi.org/10.1016/j.fertnstert.2005.12.076

15. McCluggage WG. Endometriosis-related pathology: a discussion of selected uncommon benign, premalignant and malignant lesions. Histopathology. 2020;76(1):76-92. https://doi.org/10.1111/his.13970

16. Iqbal CW, Kamath AS, Zietlow SP. Appendiceal intussusception masquerading as an ileocolic intussusception. J Gastrointest Surg. 2012;16(5):1076-7. https://doi.org/10.1007/s11605-012-1848-z

17. Salehzadeh A, Scala A, Simson JN. Appendiceal intussusception mistaken for a polyp at colonoscopy: case report and review of literature. Ann R Coll Surg Engl. 2010;92(6):W46-8. https://doi.org/10.1308/147870810X12699662981591

18. Birriel TJ, Smith E, Eyvazzadeh D. Appendiceal intussusception from endometriosis: endoscopic and laparoscopic approach. Ann R Coll Surg Engl. 2017;99(1):e1-e2. https://doi.org/10.1308/rcsann.2016.0244

19. Sriram PV, Seitz U, Soehendra N, Schroeder S. Endoscopic appendectomy in a case of appendicular intussusception due to endometriosis, mimicking a cecal polyp. Am J Gastroenterol. 2000;95(6):1594-6. https://doi.org/10.1111/j.1572-0241.2000.02108.x

20. Tavakkoli H, Sadrkabir SM, Mahzouni P. Colonoscopic diagnosis of appendiceal intussusception in a patient with intermittent abdominal pain: a case report. World J Gastroenterol. 2007;13(31):4274-7. https://doi.org/10.3748/wjg.v13.i31.4274

21. Betancourth-Alvarenga JE, Vázquez-Rueda F, MurciaPascual FJ, Ayala-Montoro J. Abdomen agudo secundario a invaginación apendicular. An Pediatr (Barc). 2015;82(1):e56-9. https://doi.org/10.1016/j.anpedi.2014.01.016

22. Suazo C, Burdiles $P$, Larach A. Invaginación apendicular. Revista Chilena de Cirugía. 2012;64(1):76-8. https://doi.org/10.4067/S0718-40262012000100013 